\title{
Hypercyclic operators with an infinite dimensional closed subspace of periodic points
}

\author{
Sophie Grivaux \\ Équipe d'Analyse \\ Université Paris 6, Case 186 \\ 4, Place Jussieu \\ 75252 Paris Cedex 05, France \\ grivaux@ccr.jussieu.fr
}

Recibido: 13 de Septiembre de 2002

Aceptado: 12 de Febrero de 2003

\begin{abstract}
Let $X$ be an infinite dimensional separable Banach space. There exists a hypercyclic operator on $X$ which is equal to the identity operator on an infinite dimensional closed subspace of $X$.
\end{abstract}

2000 Mathematics Subject Classification: 47A16.

Key words: Hypercyclic operators, chaotic operators

\section{Introduction and main results}

In this note, we will be concerned with hypercyclic and chaotic linear operators on an infinite dimensional real or complex separable Banach space $X$. If $T$ is a bounded operator on $X$, a vector $x$ of $X$ is said to be hypercyclic for $T$ if the orbit of $x$ under $T$, that is $\operatorname{Orb}(x, T)=\left\{T^{n} x, n \geq 0\right\}$, is dense in $X$. If $T$ has a hypercyclic vector, $T$ is called a hypercyclic operator. There is a link between hypercyclicity and dynamics for linear operators on a Banach space. Recall that a continuous mapping $\phi$ of a metric space $E$ is chaotic in the sense of Devaney ([8], p. 50) if it satisfies the following three conditions: it is topologically transitive (i.e. for every pair of non empty open subsets $U, V$ of $E$, there exists an integer $n$ such that $\phi^{n}(U) \cap V$ is non-void), it has a dense set of periodic points (a vector $x$ of $X$ is called periodic for $\phi$ when there exists a positive integer $n$ such that $\phi^{n} x=x$ ), and it has sensitive dependence on initial conditions (there exists a positive real number $\delta$ such that for every $x$ in $X$ and every neighborhood $V$ of $X$, there exists a vector $y$ in $V$ and a nonnegative integer 
$n$ such that $\phi^{n} x$ and $\phi^{n} y$ are more than the distance $\delta$ apart). It is shown in [3] that if $\phi$ is topologically transitive and has dense periodic points, then $\phi$ has sensitive dependence on initial conditions. In the special case where $\phi$ is a bounded operator on a separable Banach space $X$, it is shown in [9] that $\phi$ is topologically transitive if and only if it is hypercyclic. Thus $\phi$ is chaotic if and only if it is hypercyclic and has a dense set of periodic points.

A result of S. Ansari ([2]) and L. Bernal-González ([5]) states that every infinite dimensional separable Banach space supports a hypercyclic operator. It is interesting to notice here that this result cannot be extended to non-separable Banach spaces by replacing the concept of hypercyclicity by the concept of topological transitivity: a result of T. Bermudez and N.J. Kalton ([4]) implies that some non-separable spaces such as $\ell_{\infty}$ or $\mathcal{B}\left(\ell_{2}\right)$ do not support any topologically transitive operator. Another question which follows naturally from S. Ansari and L. Bernal-González result is to know whether every infinite dimensional separable Banach space supports a chaotic operator. The answer is no: J. Bonet, F. Martínez-Giménez and A. Peris proved in [6] that when $X$ is a hereditarily indecomposable Banach space with hereditarily indecomposable dual, there exists no chaotic operator on $X$ (see also [10], Section 5.3). This raises the following question regarding periodic points:

Question 1.1 [6] Does every infinite dimensional separable Banach space support a hypercyclic operator with an infinite dimensional closed subspace of periodic points?

The purpose of this note is to answer this question in the affirmative. We will prove the following stronger theorem:

Theorem 1.2. Let $X$ be an infinite dimensional separable Banach space. There exists a hypercyclic operator on $X$ which is equal to the identity operator on an infinite dimensional closed subspace of $X$.

This highlights the fact that a hypercyclic operator can be very far from having no non trivial invariant subspace. This theorem is also related to a result of [6], where it is proved that when $X$ and its dual are hereditarily indecomposable and $T$ is hypercyclic, there exists a positive integer $N$ such that every periodic vector $x$ satisfies $T^{N} x=x$. Since $T$ is hypercyclic, $T^{N}$ is hypercyclic too ([1]) and this implies that if $T$ has an infinite dimensional closed subspace $V$ of periodic points, then $T^{N}$ is hypercyclic and equal to the identity operator on this same subspace $V$.

The main step in the proof of Theorem 1.2 is Theorem 1.3, in which we construct hypercyclic perturbations of the identity operator by some kind of shift operator with infinite dimensional kernel on one of the sequence spaces $\ell_{p}, 1 \leq p<\infty$, or $c_{0}$. Theorem 1.3 is related to a result of H. Salas, who proved in [11] that when $\left(\omega_{i}\right)_{i \geq 0}$ is any bounded sequence of positive scalars and $S$ is the backward shift defined on $X$ by $S e_{0}=0$ and for every $i \geq 0, S e_{i+1}=\omega_{i} e_{i}$, then the operator $I+S$ is hypercyclic.

Theorem 1.3. Let $X$ be one of the sequence spaces $\ell_{p}, 1 \leq p<\infty$, or $c_{0}$, with canonical basis $\left(e_{i}\right)_{i \geq 0}$. Let $\left(\omega_{i}\right)_{i \geq 0}$ be any bounded sequence of positive scalars and 
consider the operator $T$ defined on $X$ by the following relations: for every $i \geq 0$,

$$
T e_{2 i}=0 \text { and } T e_{2 i+1}=\omega_{i} e_{i} .
$$

Then the operator $I+T$, where I denotes the identity operator on $X$, is hypercyclic on $X$, and it is equal to the identity operator on the closed space spanned by the vectors $e_{2 i}, i \geq 0$.

Using the notion of quasi-extension of an operator on the space $\ell_{1}$ to an operator on a general separable Banach space $X$ introduced by S. Ansari in [2], we then deduce Theorem 1.2 from Theorem 1.3.

Remark 1.4. All the hypercyclic operators constructed in Theorem 1.3 satisfy the Hypercyclicity Criterion (see for instance [7]): since $\left(\omega_{i}\right)_{i \geq 0}$ is a sequence of positive scalars, this is a straightforward consequence of Corollary 2.8 of [7].

\section{Proofs of Theorems 1.2 and 1.3}

\subsection{Proof of Theorem 1.3}

We will prove Theorem 1.3 in the Hilbert space case when $p=2$, the construction being the same in the general case.

Notation. $\left(e_{i}\right)_{i>0}$ denotes the canonical basis of $\ell_{2}$. A vector $x$ of $\ell_{2}$ is said to have finite support when it is a linear combination of finitely many basis vectors $e_{i}$, and if $x=\sum_{i>0} x_{i} e_{i}, \operatorname{supp}(x)=\left\{i \geq 0 ; x_{i} \neq 0\right\}$ denotes the support of $x$.

Let $\left(z_{k}\right)_{k \geq 1}$ be a dense sequence of vectors of $\ell_{2}$ with finite support such that for every $k \geq 1, \max \operatorname{supp}\left(z_{k}\right) \leq k$. Proceeding as in the proof of Theorem 3.3 in [11], we will construct inductively a fast increasing sequence $\left(n_{k}\right)_{k \geq 1}$ of integers and a sequence $\left(y_{k}\right)_{k \geq 1}$ of finitely supported vectors of $\ell_{2}$ such that:

(1) for every $k \geq 2, \max \operatorname{supp}\left(y_{k-1}\right)<\min \operatorname{supp}\left(y_{k}\right)$

(2) for every $k \geq 1,\left\|y_{k}\right\| \leq 2^{-(k+1)}(1+\|T\|)^{-\left(n_{k}+1\right)}$

(3) for every $k \geq 1,\left\|(I+T)^{n_{k}}\left(\sum_{j=1}^{k} y_{j}\right)-z_{k}\right\| \leq 2^{-k}$.

Then an easy computation shows that the vector $y=\sum_{k=1}^{+\infty} y_{k}$ is a hypercyclic vector for the operator $I+T$.

A crucial lemma is the following ([11], Lemma 3.2):

Lemma 2.1. Let $n$ and $r$ be positive integers. Consider the $2^{r} \times 2^{r}$ matrix $C_{n}$ defined by $C_{n}=\left(c_{i j}(n)_{1 \leq i, j \leq 2^{r}}\right)$ where $c_{i j}(n)=\left(\begin{array}{c}n \\ 2^{r}+j-i\end{array}\right)$. Let $B_{n}=\left(b_{i}(n)\right)_{1 \leq i \leq 2^{r}}$ be a column vector such that for every $i, 1 \leq i \leq 2^{r}, b_{i}(n)$ is a polynomial in $n$ of degree at most $2^{r}-i$. Then the equation $C_{n} X_{n}=B_{n}$ has a solution $X_{n}=\left(x_{i}(n)\right)_{1 \leq i<2^{r}}$ for $n$ large enough which satisfies: there exists a constant $P$ such that for every $i, 1 \leq i \leq 2^{r}$, $\left|x_{i}(n)\right| \leq \frac{P}{n^{i}}$. 
Let us first construct $y_{1}: z_{1}$ can be written as $z_{1}=z_{0}^{(1)} e_{0}+z_{1}^{(1)} e_{1}$. We will be looking for a vector $y_{1}$ of the form $y_{1}=x_{1} e_{3}+x_{2} e_{7}$. Now:

$$
\begin{aligned}
& \left\|(I+T)^{n}\left(x_{1} e_{3}+x_{2} e_{7}\right)-z_{1}\right\|=\left\|\left(x_{1}+x_{2} \omega_{3}\left(\begin{array}{l}
n \\
1
\end{array}\right)\right) e_{3}+x_{2} e_{7}\right\| \\
& +\left\|\left(x_{1} \omega_{1} \omega_{0}\left(\begin{array}{c}
n \\
2
\end{array}\right)+x_{2} \omega_{3} \omega_{1} \omega_{0}\left(\begin{array}{c}
n \\
3
\end{array}\right)-z_{0}^{(1)}\right) e_{0}+\left(x_{1} \omega_{1}\left(\begin{array}{c}
n \\
1
\end{array}\right)+x_{2} \omega_{3} \omega_{1}\left(\begin{array}{c}
n \\
2
\end{array}\right)-z_{1}^{(1)}\right) e_{1}\right\| .
\end{aligned}
$$

Since

$$
\left.\left|\begin{array}{cc}
\omega_{1} \omega_{0}\left(\begin{array}{l}
n \\
2
\end{array}\right) & \omega-3 \omega_{1} \omega_{0}\left(\begin{array}{c}
n \\
3
\end{array}\right) \\
\omega_{1}\left(\begin{array}{l}
n \\
1
\end{array}\right) & \omega_{3} \omega_{1}\left(\begin{array}{l}
n \\
2
\end{array}\right)
\end{array}\right|=\omega_{3} \omega_{1}^{2} \omega_{0} \mid \begin{array}{ll}
\left(\begin{array}{c}
n \\
2
\end{array}\right) & \left(\begin{array}{l}
n \\
3
\end{array}\right) \\
\left(\begin{array}{l}
n \\
1
\end{array}\right) & \left(\begin{array}{l}
n \\
2
\end{array}\right)
\end{array}\right),
$$

the system

$$
\left(\begin{array}{cc}
\omega_{1} \omega_{0}\left(\begin{array}{l}
n \\
2
\end{array}\right) & \omega_{3} \omega_{1} \omega_{0}\left(\begin{array}{l}
n \\
3
\end{array}\right) \\
\omega_{1}\left(\begin{array}{l}
n \\
1
\end{array}\right) & \omega_{3} \omega_{1}\left(\begin{array}{l}
n \\
2
\end{array}\right)
\end{array}\right)\left(\begin{array}{l}
x_{1} \\
x_{2}
\end{array}\right)=\left(\begin{array}{c}
z_{0}^{(1)} \\
z_{1}^{(1)}
\end{array}\right)
$$

has a solution for $n$ large enough with $\left|x_{i}(n)\right| \leq \frac{P}{n^{i}}$ for $i=1,2$. Then

$$
\left\|(I+T)^{n}\left(x_{1}(n) e_{3}+x_{2}(n) e_{7}\right)-z_{1}=\right\|\left(x_{1}(n)+x_{2}(n) \omega_{3}\left(\begin{array}{c}
n \\
1
\end{array}\right)\right) e_{3}+x_{2}(n) e_{7} \| \underset{+\infty}{=} O\left(\frac{1}{n}\right) .
$$

If $n_{1}$ is big enough and $y_{1}=x_{1}\left(n_{1}\right) e_{3}+x_{2}\left(n_{1}\right) e_{7}$, then $y_{1}$ satisfies conditions (2) and (3) for $k=1$.

Suppose now that $y_{1}, \ldots y_{k-1}, n_{1}, \ldots n_{k-1}$ have already been constructed, and let $r$ be a positive integer such that $2^{r}-1 \geq k$ and for every $i=1 \ldots k-1, \operatorname{supp}\left(y_{i}\right) \subseteq$ $\left\{0 \ldots 2^{r}-1\right\}$.

Notation. For $l \geq 0$, the sequence $\left(u_{n}^{(2 l)}\right)_{n \geq 0}$ is the sequence defined by

$$
u_{0}^{(2 l)}=2 l \text { and for } n \geq 0, u_{n+1}^{(2 l)}=2 u_{n}^{(2 l)}+1 .
$$

Let $I_{2 l}$ be the set of cardinality $2^{r}$ containing the $2^{r}$ first terms of the sequence $\left(u_{n}^{(2 l)}\right)_{n \geq 0}$ :

$$
I_{2 l}=\left\{u_{0}^{(2 l)}, u_{1}^{(2 l)}, \ldots, u_{2^{r}-1}^{(2 l)}\right\} .
$$

It is easy to see that the sets $I_{2 l}$ are disjoint. Denote by $\Pi_{2 l}$ the orthogonal projection onto the space spanned by the vectors $e_{v}, v \in I_{2 l}$.

With this notation, $T e_{u_{n+1}^{(2 l)}}=\omega_{u_{n}^{(2 l)}} e_{u_{n}^{(2 l)}}$ for every $n \geq 0$. Moreover, $\left\{u_{j}^{(2 l)}\right\}$ is the support of the vector $T^{-j} e_{u_{0}^{(2 l)}}$ for every $j \geq 1$, and

$$
e_{u_{j}^{(2 l)}}=\left(\prod_{i=0}^{j-1} \omega_{u_{i}^{(2 l)}}\right) T^{-j} e_{u_{0}^{(2 l)}} .
$$


We will be looking for a vector $y_{k}$ of the form $y_{k}=\sum_{l=0}^{s}\left(\sum_{i=1}^{2^{r}} x_{i}^{(2 l)} e_{u_{2^{r}+i-1}^{(2 l)}}\right)$ where the coordinates $x_{i}^{(2 l)}$ have to be determined. This vector $y_{k}$ must satisfy $s+1$ systems of equations: for every $l=0 \ldots s$ and for every $p=0 \ldots 2^{r}-1$,

$$
\left\langle(I+T)^{n}\left(\sum_{l=0}^{s}\left(\sum_{i=1}^{2^{r}} x_{i}^{(2 l)} e_{u_{2^{r}+i-1}^{(2 l)}}\right)+\sum_{j=1}^{k-1} y_{j}\right)-z_{k}, e_{u_{p}^{(2 l)}}\right\rangle=0 .
$$

Since the sets $I_{0}, I_{2}, \ldots, I_{2 s}$ do not overlap and since the vector space spanned by the vectors $e_{v}, v \in I_{2 l}$, is invariant by $T$, the equations above are equivalent to the following $s+1$ independent systems:

$$
\left(\mathcal{S}_{2 l}\right)\left\{\begin{array}{l}
\text { for every } p=0 \ldots 2^{r}-1, \\
\left\langle(I+T)^{n}\left(\sum_{i=1}^{2^{r}} x_{i}^{(2 l)} e_{u_{2^{r}+i-1}^{(2 l)}}\right), e_{\left.u_{p}^{(2 l)}\right\rangle}\right\rangle \\
=\left\langle\Pi_{2 l}\left(z_{k}-(I+T)^{n} \sum_{j=1}^{k-1} y_{j}\right), e_{u_{p}^{(2 l)}}\right\rangle
\end{array}\right.
$$

for $l=0 \ldots s$.

Now

$$
\begin{array}{r}
\left\langle(I+T)^{n}\left(\sum_{i=1}^{2^{r}} x_{i}^{(2 l)} e_{u_{2^{r}+i-1}^{(2 l)}}\right), e_{u_{p}^{(2 l)}}\right\rangle=\left\langle\sum_{i=1}^{2^{r}} x_{i}^{(2 l)} \sum_{j=0}^{n}\left(\begin{array}{c}
n \\
j
\end{array}\right) T^{j} e_{u_{2^{2}+i-1}^{(2 l)}}, e_{u_{p}^{(2 l)}}\right\rangle \\
=\left\langle\sum_{i=1}^{2^{r}} x_{i}^{(2 l)} \sum_{j=0}^{n}\left(\begin{array}{c}
n \\
j
\end{array}\right)\left(\prod_{m=2^{2}+i-j-1}^{2^{r}+i-2} \omega_{u_{m}^{(2 l)}}\right) e_{u_{2^{r}+i-j-1}^{(2 l)}}, e_{u_{p}^{(2 l)}}\right\rangle .
\end{array}
$$

Thus the system $\left(\mathcal{S}_{2 l}\right)$ has the form $C_{n}^{(2 l)} X_{n}^{(2 l)}=B_{n}^{(2 l)}$ with $X_{n}^{(2 l)}=\left(x_{p}^{(2 l)}(n)\right)_{1 \leq p \leq 2^{r}}$,

$$
B_{n}^{(2 l)}=\left(b_{p}^{(2 l)}(n)\right)_{1 \leq p \leq 2^{r}}=\left(\left\langle\Pi_{2 l}\left(z_{k}-(I+T)^{n} \sum_{j=1}^{k-1} y_{j}\right), e_{u_{p-1}^{(2 l)}}\right\rangle\right)_{1 \leq p \leq 2^{r}}
$$

and $C_{n}^{(2 l)}=\left(c_{i j}^{(2 l)}(n)\right)_{1 \leq i, j \leq 2^{r}}$ where $c_{i j}^{(2 l)}(n)=\left(\prod_{m=2^{r}+i-j-1}^{2^{r}+i-2} \omega_{u_{m}^{(2 l)}}\right)\left(\begin{array}{l}n \\ j\end{array}\right)$. Moreover,

$$
\operatorname{det} C_{n}^{(2 l)}=\left(\prod_{q=0}^{2^{r}-1} \prod_{m=q}^{2^{r}-1} \omega_{u_{m}^{(2 l)}}\right)\left(\prod_{q=0}^{2^{r}-1} \prod_{m=0}^{q} \omega_{u_{m}^{(2 l)}}\right) \operatorname{det} C_{n},
$$

where $C_{n}$ is the matrix of size $2^{r} \times 2^{r}$ considered in Lemma 2.1. Thus in order to apply Lemma 2.1 , we only have to check that for $p=1 \ldots 2^{r}, b_{p}^{(2 l)}(n)$ is a polynomial in $n$ of degree at most $2^{r}-p$. The projection onto $I_{2 l}$ of the vector $\sum_{j=1}^{k-1} y_{j}$ can be written as $\Pi_{2 l}\left(\sum_{j=1}^{k-1} y_{j}\right)=\sum_{i=0}^{2^{r}-1} \alpha_{i}^{(2 l)} e_{u_{i}^{(2 l)}}$, and since $\Pi_{2 l}(I+T)^{n} \sum_{j=1}^{k-1} y_{j}=$ $(I+T)^{n} \Pi_{2 l} \sum_{j=1}^{k-1} y_{j}$, this yields that

$$
\left\langle\Pi_{2 l}(I+T)^{n} \sum_{j=1}^{k-1} y_{j}, e_{u_{p-1}^{(2 l)}}\right\rangle=\sum_{i=0}^{2^{r}-1} \alpha_{i}^{(2 l)} \sum_{q=0}^{n}\left(\begin{array}{l}
n \\
q
\end{array}\right)\left\langle T^{q} e_{u_{i}^{(2 l)}}, e_{u_{p-1}^{(2 l)}}\right\rangle .
$$


Since $\left\langle T^{q} e_{u_{i}^{(2 l)}}, e_{u_{p-1}^{(2 l)}}\right\rangle$ is non-zero if and only if $q=i-p+1$, the expression above can be reduced to

$$
b_{p}^{(2 l)}(n)=\left\langle z_{k}, e_{u_{p-1}^{(2 l)}}\right\rangle-\sum_{i=0}^{2^{r}-1} \alpha_{i}^{(2 l)}\left(\begin{array}{c}
n \\
i-p+1
\end{array}\right)\left\langle T^{i-p+1} e_{u_{i}^{(2 l)},} e_{u_{p-1}^{(2 l)}}\right\rangle .
$$

This shows that $b_{p}^{(2 l)}(n)$ is a polynomial in $n$ of degree at most $2^{r}-p$. We can therefore apply Lemma 2.1 and obtain a solution $\left(x_{i}^{(2 l)}(n)\right)_{1 \leq i \leq 2^{r}}$ of the system $\left(\mathcal{S}_{2 l}\right)$ with

$$
\left|x_{i}^{(2 l)}(n)\right| \leq \frac{P^{(2 l)}}{n^{i}}
$$

for $n$ large enough, where $P^{(2 l)}$ is some constant depending only on $l$. Setting

$$
y_{k}(n)=\sum_{l=0}^{s}\left(\sum_{i=1}^{2^{r}} x_{i}^{(2 l)}(n) e_{u_{2^{r}+i-1}^{(2 l)}}\right)
$$

we have

$$
\begin{aligned}
(I+T)^{n}\left(\sum_{j=1}^{k-1} y_{j}+y_{k}(n)\right) & -z_{k}=\sum_{q=0}^{+\infty}\left\langle(I+T)^{n}\left(\sum_{j=1}^{k-1} y_{j}+y_{k}(n)\right)-z_{k}, e_{q}\right\rangle e_{q} \\
& =\sum_{q \notin I_{0} \cup I_{2} \cup \ldots \cup I_{2 s}}\left\langle(I+T)^{n}\left(\sum_{j=1}^{k-1} y_{j}+y_{k}(n)\right)-z_{k}, e_{q}\right\rangle e_{q} .
\end{aligned}
$$

Now $\sum_{j=1}^{k-1} y_{j}$ and $z_{k}$ belong to the space spanned by the vectors $e_{v}, v \in I_{0} \cup I_{2} \cup \ldots \cup$ $I_{2 s}$, which is invariant by $(I+T)^{n}$, and thus the last equality becomes:

$$
\begin{aligned}
(I+T)^{n}\left(\sum_{j=1}^{k-1} y_{j}+y_{k}(n)\right) & -z_{k}=\sum_{q \notin I_{0} \cup I_{2} \cup \ldots \cup I_{2 s}}\left\langle(I+T)^{n}\left(y_{k}(n)\right), e_{q}\right\rangle e_{q} \\
& =\sum_{l=0}^{s} \sum_{i=1}^{2^{r}} x_{i}^{(2 l)}(n) \sum_{q \notin I_{0} \cup I_{2} \cup \ldots \cup I_{2 s}} \sum_{p=0}^{n}\left(\begin{array}{l}
n \\
p
\end{array}\right)\left\langle T^{p} e_{u_{2^{2}+i-1}^{(2 l)}}, e_{q}\right\rangle e_{q} .
\end{aligned}
$$

Since $q$ does not belong to $I_{2 l},\left\langle T^{p} e_{u_{2^{r}+i-1}^{(2 l)}}, e_{q}\right\rangle$ is zero as soon as $2^{r}+i-1-p<2^{r}$, i.e. $i-1<p$, and we obtain:

$(I+T)^{n}\left(\sum_{j=1}^{k-1} y_{j}+y_{k}(n)\right)-z_{k}=\sum_{l=0}^{s} \sum_{i=1}^{2^{r}} x_{i}^{(2 l)}(n) \sum_{p=0}^{i-1}\left(\begin{array}{c}n \\ p\end{array}\right) \sum_{q \notin I_{0} \cup I_{2} \cup \ldots \cup I_{2 s}}\left\langle T^{p} e_{u_{2}^{2 l}+i-1}, e_{q}\right\rangle e_{q}$ 
and

$$
\left\|(I+T)^{n}\left(\sum_{j=1}^{k-1} y_{j}+y_{k}(n)\right)-z_{k}\right\| \underset{+\infty}{=} O\left(\sum_{i=1}^{2^{r}}\left|x_{i}^{(2 l)}(n)\right| \sum_{j=0}^{i-1}\left(\begin{array}{l}
n \\
j
\end{array}\right)\right) \underset{+\infty}{=} O\left(\frac{1}{n}\right) .
$$

Taking $n_{k}$ large enough and $y_{k}=y_{k}\left(n_{k}\right)$ yields (1), (2) and (3). This terminates the proof of Theorem 1.3.

\subsection{Proof of Theorem 1.2}

This is a mere rewriting of the arguments of [2]. If $\left(x_{i}, x_{i}^{*}\right)_{i \geq 0}$ is a bounded biorthogonal system of the Banach space $X$ and if $\omega_{i}, i \geq 0$, are positive numbers such that $\sum_{i \geq 0}\left\|x_{2 i+1}^{*}|| \omega_{i}\right\| x_{i} \|<+\infty$, the operator

$$
\widetilde{T}(x)=\sum_{i \geq 0} x_{2 i+1}^{*}(x) \omega_{i} x_{i}
$$

is a nuclear operator on $X$. If $T$ is defined on the space $\ell_{1}$ with canonical basis $\left(e_{i}\right)_{i \geq 0}$ by $T e_{2 i}=0$ and $T e_{2 i+1}=\omega_{i} e_{i}$ for $i \geq 0$, then $I+\widetilde{T}$ is a quasi-extension of $I+T$ in the sense of [2]. Since $I+T$ is hypercyclic on $\ell_{1}, I+\widetilde{T}$ is hypercyclic on $X$, and $I+\widetilde{T}$ is equal to the identity on the infinite dimensional closed subspace spanned by the vectors $x_{2 i}, i \geq 0$. Theorem 1.2 is proved.

\section{References}

[1] S.I. Ansari, Hypercyclic and cyclic vectors, J. Func. Anal., 128 (1995), pp 374-383.

[2] S.I. Ansari, Existence of hypercyclic operators on topological vector spaces, J. Func. Anal., 148 (1997), pp 384-390.

[3] J. Banks, J. Brooks, G. Cairns, G. Davis, P. Stacey, On Devaney's definition of chaos, Amer. Math. Monthly, 99 (1992), pp 332-334.

[4] T. Bermudez, N.J. Kalton, The range of operators on Von Neumann algebras, Proc. Amer. Math. Soc., 130 (2002), pp 1447-1455.

[5] L. Bernal-González, On hypercyclic operators on Banach spaces, Proc. Amer. Math. Soc., 127 (1999), pp 1003-1010.

[6] J. Bonet, F. Martínez-Giménez, A. Peris, A Banach space with no chaotic operator, Bull. London Math. Soc., 33 (2001), pp 196-198.

[7] J. Bès, A. Peris, Hereditarily hypercyclic operators, J. Func. Anal., 167 (1999), pp 94-112.

[8] R.L. Devaney, An Introduction to Chaotic Dynamical Systems, 2nd ed., AddisonWesley, Reading, MA, 1989.

[9] G. Godefroy, J.H. Shapiro, Operators with dense, invariant, cyclic vector manifolds, J. Func. Anal., 98 (1991), pp 229-269. 
[10] S. Grivaux, Sums of hypercyclic operators, to appear in J. Func. Anal..

[11] H. Salas, Hypercyclic weighted shifts, Trans. Amer. Math. Soc., 347 (1995), pp 9931004. 